\title{
2 Untersuchungsdesign und methodisches Vorgehen
}

Sibylle Steinmann, Christian Brühwiler und Erich Ramseier

\begin{abstract}
Dieses Kapitel gibt einen Überblick zum Untersuchungsdesign und zum methodischen Vorgehen. Die nationale und internationale Stichprobe und Stichprobenziehung werden beschrieben und dargestellt. Die Entwicklung der Test-Instrumente wird erläutert und die standardisierte Durchführung der Erhebung inklusive der Qualitätssicherung wiedergegeben. Das Vorgehen der unterschiedlichen Skalierungsarten für die Daten zur fachlichen Leistung zu den Lerngelegenheiten und Überzeugungen wird dargelegt.
\end{abstract}

\subsection{Population und Stichprobe ${ }^{1}$}

Die IEA (International Association for the Evaluation of Educational Achievement) verfolgt mit TEDS-M (Teacher Education and Development Study in Mathematics) die Frage nach der Wirksamkeit der Lehrerbildung. In dieser ersten international vergleichenden Studie zur Lehrerbildung werden die Ausbildungsinstitutionen und die angehenden Lehrpersonen aus insgesamt 17 Ländern aus Nord- und Südamerika, Afrika, Europa und Asien in den Fokus genommen. Die Schweiz ist in der Studie ausschliesslich mit der Lehrerbildung der Deutschschweiz vertreten. Die Bestimmung der Population, die Ziehung der Stichproben und die Durchführung der Erhebungen wurden in allen Ländern entsprechend den Vorgaben der IEA durchgeführt (siehe Tatto et al., 2012).

1 Genauere Informationen zum Samplingverfahren und zur internationalen Stichprobenzusammensetzung sind dem internationalen TEDS-M Bericht (siehe Dumais \& Meinck, 2013b; Tatto et al., 2012) zu entnehmen. 
Teilnehmende Länder

Botswana, Chile, Deutschland, Georgien, Kanada ${ }^{2}$, Malaysia, Norwegen, Oman (nur Sekundarstufe I), Philippinen, Polen, Russland, Schweiz (nur deutschsprachiger Teil), Singapur, Spanien (nur Primarstufe), Taiwan, Thailand, USA ${ }^{3}$

\section{Zielgruppen von TEDS-M}

Für die Erhebungen im Rahmen von TEDS-M sind entsprechend dem Mehrebenenmodell (vgl. Kapitel 1) die drei Zielgruppen Institution bzw. Ausbildungsgänge, Dozierende und Studierende bzw. angehende Lehrpersonen bestimmt worden. Alle drei Zielgruppen sind auf Ausbildungen eingegrenzt, die für das Unterrichten von Mathematik auf mindestens einer Klassenstufe von 1-8 berechtigen. Jede Zielgruppe ist mit je eigenen Erhebungsinstrumenten untersucht worden.

Auf der institutionellen Ebene befinden sich die Lehrerausbildungsinstitutionen, welche in ihre Ausbildungsgänge für angehende Primar- und Sekundarlehrpersonen unterteilt sind. Die Bestimmung der Institutionen in der Deutschschweiz und die Unterteilung in Ausbildungseinheiten wurde gemäss der institutionellen Strukturen ${ }^{4}$ vorgenommen (Tabelle 2.1). So wird beispielsweise die Pädagogische Hochschule der FHNW mit den drei Institutionen „Vorschul- und Primarstufe“, „Primarstufe“" und „Sekundarstufe I“ dargestellt. Erst innerhalb dieser drei Institutionen wird die Unterteilung nach den Standorten (Ausbildungseinheit) vorgenommen. Anders verhält es sich beispielsweise bei der PHZ Zentralschweiz: Die drei Standorte (Luzern, Schwyz und Zug) verstehen sich als eigene Institutionen, welche in die jeweiligen Studiengänge (Kindergarten-Unterstufe, Primarstufe und Sekundarstufe I) unterteilt sind.

2 Kanada scheidet für die internationalen Vergleiche der angehenden Lehrpersonen wegen einer zu geringen Rücklaufquote aus.

3 In den USA sind nur Hochschulen in staatlicher Trägerschaft untersucht worden.

4 Für die Studie sind die institutionellen Gegebenheiten zum Erhebungszeitpunkt im Frühjahr 2008 massgebend. 


\begin{tabular}{|c|c|c|c|}
\hline Institution & Ausbildungsgang & 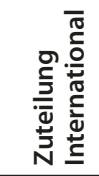 & 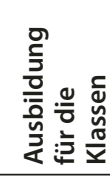 \\
\hline \multirow{2}{*}{ PH Freiburg (HP) } & PH Freiburg Kindergarten - Unterstufe & PS & KG bis 2 \\
\hline & PH Freiburg Primarstufe & PS & 3 bis 6 \\
\hline PH Graubünden (PHGR) & PH Graubünden Primarstufe & PS & 2 bis 6 \\
\hline \multirow{3}{*}{ PH St.Gallen (PHSG) } & PH Rorschach Kindergarten - Unterstufe & PS & KG bis 3 \\
\hline & PH Rorschach Primarstufe & PS & 1 bis 6 \\
\hline & PH St. Gallen Sekundarstufe I & Sek & 7 bis 9 \\
\hline PH Thurgau (PHTG) & PH Thurgau Primarstufe & PS & 1 bis 6 \\
\hline PH Schaffhausen (PHSH) & PH Schaffhausen Primarstufe & PS & 1 bis 6 \\
\hline \multirow{3}{*}{ PH Bern } & PH Bern Institut Vorschulstufe und Primarstufe & PS & KG bis 6 \\
\hline & $\begin{array}{l}\text { PH Bern NMS, Privates Institut Vorschulstufe } \\
\text { und Primarstufe }\end{array}$ & PS & KG bis 6 \\
\hline & PH Bern Sekundarstufe I & Sek & 7 bis 9 \\
\hline \multirow{2}{*}{$\begin{array}{l}\text { PH der Fachhochschule } \\
\text { Nordwestschweiz (FHNW): } \\
\text { Institut Vorschul- und Unterstufe }\end{array}$} & PH der FHNW - Liestal Unterstufe & PS & KG bis 3 \\
\hline & PH der FHNW - Solothurn Unterstufe & PS & KG bis 2 \\
\hline \multirow{3}{*}{$\begin{array}{l}\text { PH der Fachhochschule } \\
\text { Nordwestschweiz (FHNW): } \\
\text { Institut Primarstufe }\end{array}$} & PH der FHNW - Liestal Primarstufe & PS & 1 bis 6 \\
\hline & PH der FHNW - Zofingen Primarstufe & PS & 1 bis 6 \\
\hline & PH der FHNW - Solothurn Primarstufe & PS & 3 bis 6 \\
\hline \multirow{2}{*}{$\begin{array}{l}\text { PH der Fachhochschule } \\
\text { Nordwestschweiz (FHNW): } \\
\text { Institut Sekundarstufe I } \\
\end{array}$} & PH der FHNW - Aarau Sekundarstufe I & Sek & 5 bis 9 \\
\hline & PH der FHNW - Basel Sekundarstufe I & Sek & 5 bis 9 \\
\hline $\mathrm{PH}$ Wallis (PHVS) & PH Wallis - Standort Brig & PS & KG bis 6 \\
\hline \multirow{4}{*}{$\begin{array}{l}\text { PH Zentralschweiz Luzern } \\
\text { (PHZ Luzern) }\end{array}$} & PHZ Luzern - Kindergarten - Unterstufe & PS & KG bis 2 \\
\hline & PHZ Luzern - Primarstufe & PS & 1 bis 6 \\
\hline & PHZ Luzern - Sekundarstufe SR & Sek & 7 bis 9 \\
\hline & PHZ Luzern - Sekundarstufe SL & Sek & 7 bis 9 \\
\hline \multirow{2}{*}{$\begin{array}{l}\text { PH Zentralschweiz Schwyz } \\
\text { (PHZ Schwyz) }\end{array}$} & PHZ Schwyz - Kindergarten - Unterstufe & PS & KG bis 2 \\
\hline & PHZ Schwyz - Primarstufe & PS & 1 bis 6 \\
\hline \multirow{2}{*}{ PH Zentralschweiz Zug (PHZ Zug) } & $\mathrm{PHZZug}-\mathrm{KGU}$ & PS & KG bis 2 \\
\hline & PHZ Zug - Primarstufe & PS & 1 bis 6 \\
\hline \multirow{2}{*}{$\mathrm{PH}$ Zürich (PHZH) } & PH Zürich (PHZH) - Primarstufe & PS & 1 bis 6 \\
\hline & PH Zürich (PHZH) - Sek I & Sek & 7 bis 9 \\
\hline Seminar Unterstrass Zürich & Seminar Unterstrass Zürich (PHZH) & PS & 1 bis 6 \\
\hline $\begin{array}{l}\text { Universität Freiburg - Dep. } \\
\text { Erziehungswissenschaften }\end{array}$ & $\begin{array}{l}\text { Abteilung für Lehrerinnen- und Lehrerbildung } \\
\text { für die Sekundarstufe I }\end{array}$ & Sek & 7 bis 9 \\
\hline
\end{tabular}


In der Zielgruppe der Dozierenden werden entsprechend der Konzeption der Studie alle Dozierenden erfasst, die die angehenden Lehrpersonen in den Bereichen der Mathematik/Mathematikdidaktik oder der Erziehungswissenschaft/allgemeinen Didaktik unterrichten (Tabelle 2.2). International entstanden so drei Gruppen von Dozierenden: Dozierende der Mathematik/ Mathematikdidaktik, Dozierende der Erziehungswissenschaften und Dozierende, welche sowohl im Bereich der Mathematik als auch der Erziehungswissenschaften unterrichten. In der Deutschschweiz konnten nur die ersten beiden Gruppen gebildet werden.

Die Stichprobe der angehenden Lehrpersonen umfasst die Studierenden im letzten Ausbildungsjahr mit dem Ziel der Unterrichtsberechtigung für die Primar- oder Sekundarstufe I mit dem Unterrichtsfach Mathematik.

Die Ausbildung für Primarlehrpersonen erfolgt in der Schweiz im Hinblick auf verschiedene Unterrichtsstufen. Ausbildungsgänge für Lehrpersonen mit Unterrichtsberechtigung für Kindergarten bis 2. Klasse und Kindergarten bis 3. Klasse werden in der Darstellung der Ergebnisse zum Ausbildungstyp Untere Primarstufe $(1-4)^{5}$ zusammengefasst. Die Ausbildungsgänge mit der Zielstufe Kindergarten bis 6. Klasse, 1. bis 6. Klasse und 3. bis 6. Klasse werden als Primarstufe (1-6) bezeichnet. Ausbildungsgänge, welche Lehrpersonen nur für den Kindergarten ausbilden, gehören nicht zur Zielgruppe von TEDS-M.

Die TEDS-M Zielgruppe der angehenden Lehrpersonen für die Sekundarstufe I umfasst alle Studierenden, welche die Lehrberechtigung für das Fach Mathematik auf der Sekundarstufe I erwerben. In der Deutschschweiz lehren hauptsächlich Personen mit Sekundarlehrdiplom I die Klassen 7 bis 9. Insbesondere an Langzeitgymnasien werden Schülerinnen und Schüler in den Klassen 7 bis 9 auch von Lehrpersonen mit Sekundarlehrdiplom II unterrichtet. In der Deutschschweiz konnten die angehenden Lehrpersonen mit einem angestrebten Diplom für die Sekundarstufe II jedoch nicht in die Studie integriert werden, da an den Universitäten kaum Informationen vorliegen, welche Studierenden kurz vor Abschluss des Studiums stehen. Für die Deutschschweiz beschränkt sich die Stichprobe deshalb auf die angehenden Lehrpersonen mit einem Lehrdiplom für die Sekundarstufe I mit dem Unterrichtsfach Mathematik.

In verschiedenen Kapiteln werden Ergebnisse auch nach einzelnen Ausbildungsgängen der Deutschschweiz dargestellt. Aus Gründen der Anonymisierung werden keine Institutionsbezeichnungen kenntlich gemacht, stattdessen wird ein neutrales Kürzel, bestehend aus einem Buchstaben und einer Ziffer, verwendet. Dabei steht „U“ für Untere Primarstufe (1-4), P für Primarstufe

5 International umfasst dieser Ausbildungstyp alle Ausbildungsgänge, die angehende Lehrpersonen für eine Tätigkeit auf der Primarstufe bis maximal zur vierten Klasse vorbereiten. Daraus folgt die ergänzende Bezeichnung (1-4). 
(1-6) und S für Sekundarstufe I. Dieses Kürzel bezeichnet über alle Kapitel dieses Bandes hinweg jeweils denselben Ausbildungsgang.

Im Gegensatz zur Schweiz sind international in vielen Ländern Ausbildungsgänge einbezogen, die auch zum Unterricht auf der Sekundarstufe II berechtigen (siehe Tatto et al., 2012, S. 280). Dies ist insbesondere bei der Interpretation der Ergebnisse im internationalen Vergleich zu berücksichtigen. Um auf diese Unterscheidung hinzuweisen, wird deshalb die Bezeichnung „Sekundarstufe I" verwendet, wenn nur Ausbildungsgänge bzw. -typen mit angestrebter Unterrichtsberechtigung bis maximal Klassenstufe 10 gemeint sind. Die Bezeichnung „Sekundarstufe“ umfasst dagegen auch Ausbildungsgänge bzw. -typen mit angestrebter Unterrichtsberechtigung bis Klassenstufe 11 bzw. 12.

\section{Beschreibung der Stichprobenziehung mit Fokus auf die Deutschschweiz ${ }^{6}$}

TEDS-M verfolgt ein mehrstufiges stratifiziertes Stichprobendesign mit randomisierten Ziehungen in den oben beschriebenen Zielgruppen. Das Design verlangt pro Land und Level (Primarstufe und Sekundarstufe) im Minimum eine Stichprobe von 50 Institutionen und von 30 Dozierenden pro ausgewählter Institution und Fach. Die Stichproben der Studierenden innerhalb der gewählten Institutionen müssen so gross sein, dass sie zusammen mindestens gleichwertig zu einer reinen Zufallsstichprobe von 400 Studierenden aus der Gesamtpopulation sind. Dieses Design musste jeweils auf die konkrete Situation in den einzelnen Ländern abgestimmt werden (Dumais \& Meinck, 2013b). Da die gesamte Deutschschweiz weniger als 50 Institutionen aufweist, wurde eine Vollerhebung auf der Ebene der Institutionen, Dozierenden und Studierenden durchgeführt.

Alle 16 Deutschschweizer Lehrerbildungsinstitutionen haben sich an der Erhebung beteiligt. Von den 23 Ausbildungsgängen der Primarstufe haben 21 und von den acht Sekundarstufenausbildungsgängen sieben den Institutionsfragebogen ausgefüllt (Tabelle 2.2).

Bei den Dozierenden der Mathematik/Mathematikdidaktik entspricht der Rücklauf von 52 einer Teilnahmequote von 66\%. 33.3\% dieser Dozierenden sind weiblich, 66.6\% sind männlich. Bei den Dozierenden der Erziehungswissenschaft wurde ein Rücklauf von $50 \%$ erzielt. Der Anteil der weiblichen Dozierenden liegt bei diesen Dozierenden mit 40.5\% etwas höher.

Bei den angehenden Lehrpersonen haben bei der Primarstufe 75\% (936 Personen) teilgenommen. Die Studierenden sind am Ende der Ausbildung im Durchschnitt 23.7 Jahre ( $\mathrm{SD}=3.8)$. Die jüngste Person ist 20 Jahre, die älteste 53 Jahre alt. Die grosse Mehrheit (85\%) der Studierenden sind weiblich.

Der Rücklauf bei den Studierenden für die Sekundarstufe liegt bei $80 \%$ (141 Personen). Die angehenden Lehrpersonen sind beim Abschluss ihrer Aus-

6 Die Darstellung der internationalen Stichprobe findet sich in Tatto, 2012. 
bildung im Schnitt 26.2 Jahre ( $\mathrm{SD}=4.2)$ alt. Die jüngste angehende Sekundarlehrperson ist 21 Jahre, die älteste 46 Jahre alt.

Tabelle 2.2: Übersicht zu den Stichproben in der Deutschschweiz

\begin{tabular}{|c|c|c|}
\hline & Population* & $\begin{array}{l}\text { realisierte } \\
\text { Stichprobe }\end{array}$ \\
\hline Ausbildungsgänge der Primarstufe & 23 & 21 \\
\hline Ausbildungsgänge der Sekundarstufe I & 8 & 7 \\
\hline Dozierende & 416 & 220 \\
\hline Dozierende der Mathematik oder Mathematikdidaktik & 77 & 52 \\
\hline Dozierende der Erziehungswissenschaft oder allg. Didaktik & 338 & 168 \\
\hline Angehende Primarlehrpersonen & 1252 & 936 \\
\hline Untere Primarstufe (1-4) & 160 & 121 \\
\hline Primarstufe (1-6) & 1092 & 815 \\
\hline Angehende Lehrpersonen der Sekundarstufe I & 177 & 141 \\
\hline
\end{tabular}

* Die hier angegebene Population entspricht der gewichteten Population. Für Detailinformationen siehe Dumais \& Meinck 2013b.

\subsection{Entwicklung der Instrumente}

Ein internationales Expertengremium hat u.a. aufgrund von Vorarbeiten in der MT-21-Studie, einer Untersuchung für angehende Mathematiklehrpersonen in sechs Ländern (Blömeke, Kaiser \& Lehmann, 2008; Schmidt et al., 2007), in mehreren Schritten Testitems entwickelt und für TEDS-M zusammengestellt. Die Items müssen den Anforderungen nach Klarheit, Korrektheit, Beachtung kultureller Unterschiede, Klassifikation nach Subgruppen und der Relevanz für die Lehrerausbildung gerecht werden. Eine besondere Herausforderung stellte durch die Internationalität der TEDS-M-Teilnehmerländer die Beachtung der kulturellen Unterschiede dar, was sich beispielsweise in der Sicherstellung der Inhaltsvalidität zeigte (Blömeke, 2011). Nach einem Pilottest im Juni 2006 fand im Frühling 2007 der Feldtest statt. Nach jeder dieser Testphasen verfeinerte und modifizierte das Expertengremium die Items weiter (Tatto et al., 2008, S. 35ff.). Die so entstandenen Instrumente und Skalen werden in den einzelnen Kapiteln dieses Buches detailliert beschrieben. Zur Auswertung der Testinstrumente, speziell der offenen Aufgabenstellungen, wurde ein umfangreiches Scoring-Handbuch entwickelt, welches in zwei Trainingssessions unter Teilnahme aller Länder implementiert wurde. 


\subsection{Durchführung der Erhebungen in der Deutschschweiz}

Die Erhebungen wurden in der Schweiz zwischen März und Mai 2008 entsprechend den standardisierten Vorgaben der IEA durchgeführt. Die Koordination innerhalb der Lehrerbildungsinstitutionen erfolgte durch eigens für diese Aufgabe eingesetzte Institutionskoordinatorinnen und -koordinatoren.

Auf nationaler Ebene entstand ein Country-Report, welcher den Kontext und die Rahmenbedingungen der schweizerischen Lehrerausbildung beschreibt (siehe Brandt et al., 2009).

Innerhalb der Institutionen wurde nach Möglichkeit mit den Ausbildungsgangleitungen ein zweistündiges Interview durchgeführt, um einen Fragebogen zur Institution auszufüllen. Einige Ausbildungsgangsleitungen haben den Fragebogen selbstständig ausgefüllt. Der Fragebogen umfasst die Themen Ausbildungsprogrammbeschreibung, Hintergrund der Studierenden, Auswahlverfahren der Studierenden, Inhalt des Ausbildungsprogramms, Ausbildungsverantwortung und Standards, Stellenbesetzungen, Ausbildungskosten und Überlegungen zum Ausbildungsprogramm.

Zusätzlich zu den Informationen der Ausbildungsgangsleitungen hat TEDS-M Daten über Lehrpläne und Modulbeschreibungen der Institutionen gesammelt, die für eine Curriculumanalyse verwendet wurden (vgl. Kapitel 6).

Die Dozierenden füllten einen Fragebogen zu folgenden Themen aus: akademischer Hintergrund, Berufserfahrung als Lehrpersonen und als Forschende, Ausbildung in der Schulpraxis, angebotene Lerngelegenheiten (Opportunities to Learn - OTL), Kohärenz der Lehrerausbildung und Überzeugungen zur Mathematik (vgl. Kapitel 5).

Die Befragung und Testung der angehenden Lehrpersonen wurden in den Lehrerausbildungsinstitutionen unter der Anleitung von spezifisch ausgebildeten Testadministratorinnen und -administratoren durchgeführt. Der internationale Fragebogen umfasst vier Teile (Teil A bis D; Tabelle 2.3). In der Deutschschweiz wurden zusätzlich zum internationalen Kernteil weitere Instrumente eingesetzt. Dabei handelt es sich um eine internationale und nationale Option zur Erfassung erziehungswissenschaftlichen Wissens, die mit weiteren berufsrelevanten Fragen (z.B. zu motivationalen Aspekten) ergänzt wurden. Die Durchführung der gesamten Erhebung beanspruchte bei den Studierenden insgesamt drei Stunden. 
Tabelle 2.3: Testablauf

\begin{tabular}{ll}
\hline $\begin{array}{l}\text { Einführung: Vorbereitung auf die Erhebung, Durchlesen der } \\
\text { Anleitungen usw. }\end{array}$ & 10 Minuten \\
\hline Teil A: Demografische Angaben & 5 Minuten \\
\hline Teil B: Lerngelegenheiten in der Ausbildung & 15 Minuten \\
\hline Teil C: Mathematik- und Mathematikdidaktik-Test & 60 Minuten \\
\hline Teil D: Überzeugungen zu Mathematik und Unterrichten & 10 Minuten \\
\hline PAUSE & 20 Minuten \\
\hline Teil E: Erziehungswissenschaften I (internationale Option) & 30 Minuten \\
\hline Teil F: Erziehungswissenschaften II (nationale Option) & 30 Minuten \\
\hline
\end{tabular}

\subsection{Qualitätssicherung}

Die Qualität der Durchführung in den Ländern wird von der IEA durch mehrere standardisierte Massnahmen gesichert. So folgte die Erstellung und Anpassung der Testinstrumente einem genau festgelegten Vorgehen, dessen einzelne Schritte (erste Übersetzung, kontrollierte Übersetzung, Layout) durch die IEA überwacht wurden. Die Erhebungen erfolgten gemäss einem standardisierten Ablauf. In jedem Land wurde eine Person bestimmt, die das Einhalten der international vorgegebenen Richtlinien bei der Durchführung der Erhebungen sowie die eingesetzten Testinstrumente kontrollierte. Diese Person wurde in einer zweitägigen Ausbildung durch die IEA auf ihre Aufgabe vorbereitet.

\section{$2.5 \quad$ Skalierung}

\subsubsection{Skalierung der fachlichen Leistungen}

Die Testitems zum mathematischen und mathematikdidaktischen Wissen umfassen geschlossene (Multiple choice) und offene Antwortformate.

Für die Auswertung mussten die offenen Antwortformate in einem Scoring-Verfahren beurteilt und bewertet werden. Damit sichergestellt werden konnte, dass in allen teilnehmenden Ländern die Antworten nach denselben Richtlinien gescort wurden, wurden alle Scorerinnen und Scorer in internationalen Workshops in die Arbeit eingeführt und trainiert. Innerhalb der Länder wurde eine bestimmte Anzahl von zufällig ausgewählten Testheften durch zwei verschiedene Scorerinnen bzw. Scorer bewertet. So konnte über ein Inter- 
rater-Verfahren erfasst werden, dass die Beurteilungen in ausreichendem Masse übereinstimmen.

Damit das mathematische und mathematikdidaktische Wissen breit getestet werden konnte, ohne dass die Testzeit zu lange und damit die Testbelastung für die angehenden Lehrpersonen zu gross ist, wurde für die Durchführung der Leistungstests ein sogenanntes Multi-Matrix-Design (z.B. Adams \& Wu, 2002) gewählt. Die Grundidee besteht darin, dass einzelne Aufgabenblökke systematisch zu verschiedenen Testheften zusammengestellt und diese zufällig auf die Testpersonen aufgeteilt werden. Dadurch mussten die angehenden Lehrpersonen je nur eine Teilmenge des gesamten Aufgabenpools lösen. Damit sich solche Datensätze mit zufällig verteilten fehlenden Werten trotzdem auf einer gemeinsamen Skala abbilden lassen, muss die Skalierung mittels Verfahren der probabilistischen Testtheorie (Raschskalierung bzw. ItemResponse-Theorie; Rasch, 1960; Masters, 1982) erfolgen. ${ }^{7}$

Die so ermittelten Testleistungen wurden sowohl für das mathematische Wissen wie auch für das mathematikdidaktische Wissen so transformiert, dass der Mittelwert über alle Länder hinweg 500 Punkte und die Standardabweichung 100 Punkte betragen. Somit liegen rund zwei Drittel der Leistungen zwischen 400 und 600 Punkten sowie 95\% zwischen 300 und 700 Punkten. Aus diesem Vorgehen folgt auch, dass die beiden Testwerte für Mathematik und Mathematikdidaktik nicht direkt miteinander vergleichbar sind.

Die Rasch-Skalierung hat den Vorteil, dass neben den Personen auch die Testaufgaben auf der Skala eingeordnet werden können, und zwar dort, wo Personen mit genau der entsprechenden Fähigkeit eine bestimmte Wahrscheinlichkeit (z.B. 70\%) haben, sie zu lösen. Kennt man die Positionen einer Person und einer Aufgabe auf der Skala, so kann für jedes Aufgaben-Personen-Paar angegeben werden, wie gross die Wahrscheinlichkeit ist, dass die Person diese Aufgabe lösen kann. Damit kann die Skala bzw. können Referenzwerte auf der Skala inhaltlich durch die Art der Aufgaben charakterisiert werden, die Personen mit einem Testergebnis im Bereich eines Referenzwerts mit hoher Sicherheit (70\% oder mehr) bzw. eher nicht (50\% oder weniger) lösen können. Auf diese Art wurden für das mathematische Wissen zwei Referenzwerte bestimmt (Tatto et al., 2012), mit deren Hilfe drei Leistungsniveaus der mathematischen Leistungsfähigkeit der angehenden Lehrpersonen gebildet werden können (vgl. Kapitel 10). Für das mathematikdidaktische Wissen wurde nur ein Referenzwert ermittelt, was die Unterteilung in zwei Leistungsniveaus ermöglicht (vgl. Kapitel 11).

7 Detailliertere Informationen zum Testdesign und zur Skalierung der Leistungstests finden sich im technischen Bericht von TEDS-M (Tatto et al., 2012) sowie in Blömeke, Lehmann und Suhl (2010). 


\subsubsection{Skalierung der Lerngelegenheiten und der Überzeugungen}

Die Items für die Skalen zu den Lernmöglichkeiten und Überzeugungen enthalten unterschiedliche Antwortmöglichkeiten mit zwei bis sechs Stufen. Aufgrund theoretischer Überlegungen, Ergebnissen aus früheren Studien und mithilfe explorativer Faktorenanalysen wurden Skalen gebildet, die anschliessend mittels konfirmatorischer Faktorenanalysen über die Länder hinweg auf Vergleichbarkeit getestet wurden (Tatto et al., 2012).

Die Skalierung erfolgte mit verschiedenen Verfahren. Die OTL-Skalen, die auf dichotomen Items beruhen, werden mittels Summenscores abgebildet. Die anderen Skalen aus dem internationalen Teil von TEDS-M, die auf vierbis sechsstufigen Likert-Skalen basieren, wurden raschskaliert. Alle Skalen zu den Lerngelegenheiten und den Überzeugungen, die aufgrund einer RaschSkalierung gebildet wurden, sind so transformiert, dass der jeweilige theoretische Skalenmittelpunkt (was einer neutralen Position entspricht) bei 10 liegt (Blömeke, Lehmann \& Suhl, 2010).

Die in der Schweiz im Rahmen der deutschschweizerischen Ergänzungsstudie zusätzlich eingesetzten Skalen (z.B. zu den motivationalen Orientierungen) wurden likert-skaliert. Die Skalenwerte wurden gebildet, indem jeweils der Mittelwert der einzelnen Items berechnet wurde.

\subsection{Statistische Auswertung}

Die statistische Auswertung der Stichprobendaten muss auf die komplexe Struktur der Stichproben in den einzelnen Ländern abgestimmt werden, um zu repräsentativen Aussagen über die Populationen zu kommen. Dazu werden erstens die Daten der einzelnen Personen mit Gewichten multipliziert, die angeben, wie viele Personen der Population eine bestimmte Person der Stichprobe repräsentieren. Diese Gewichte reflektieren einerseits die Wahrscheinlichkeit, mit der eine Person für die Stichprobe ausgewählt wurde. Andererseits berücksichtigen sie auch, für wie viele ausgewählte Personen einer Institution Daten fehlen (z.B. wegen krankheitsbedingter Abwesenheit am Testtag). Da in der Schweiz eine Vollerhebung durchgeführt wurde, ist hier nur dieser letztere Effekt relevant. Dank der Gewichte können somit Populationsstatistiken wie z.B. Mittelwerte korrekt geschätzt werden. Zweitens muss auch bei der Bestimmung der Genauigkeit einer Schätzung die Stichprobenstruktur berücksichtigt werden.

Jeder Schluss von einer Stichprobe auf eine Population ist mit einer Unsicherheit behaftet, da die Zusammensetzung der Stichprobe von jener der Population zufällig abweichen kann. Der Standardfehler und das daraus abgeleitete Konfidenzintervall geben an, mit welcher Abweichung zwischen einer Stati- 
stik (z.B. Mittelwert) der Stichprobe und der Population zu rechnen ist. Unterscheiden sich zwei Stichproben (z.B. zweier Länder oder aufgeteilt nach Geschlecht) mehr, als aufgrund der Standardfehler mit einer Wahrscheinlichkeit von $5 \%$ erwartet werden muss, so wird der Unterschied als statistisch signifikant bezeichnet.

Angesichts der Gewichtung und der komplexen Struktur der Stichproben können die gängigen Verfahren zur Bestimmung des Standardfehlers nicht benutzt werden, da diese nur für einfache Zufallsstichproben gelten. Stattdessen werden in TEDS-M wie in internationalen Vergleichsstudien üblich Resampling-Verfahren eingesetzt. Bei diesen Verfahren werden unter Berücksichtigung der Stichprobenstruktur wiederholt Teilstichproben gebildet. Aus der Variation einer Statistik (z.B. Mittelwert) zwischen diesen Teilstichproben kann auf den Standardfehler der Statistik in der ganzen Stichprobe geschlossen werden. Konkret wird in TEDS-M das BBR-Verfahren (balanced repeated replication) nach Fay eingesetzt (genauere Angaben dazu in Dumais \& Meinck, 2013a).

In der Deutschschweiz wurde eine Vollerhebung durchgeführt. Wenn man davon absieht, dass es zu Datenausfällen kam, kann man argumentieren, dass sich die Bestimmung eines Standardfehlers erübrigt, da ja eben die ganze Population erfasst wurde und somit eine Statistik wie der Mittelwert für z.B. die männlichen bzw. weiblichen Studierenden oder die einzelnen Institutionen (fast) fehlerfrei vorliegt. Dennoch ist die Bestimmung von Standardfehlern und statistischer Signifikanz sinnvoll. Man möchte schliesslich den gefundenen Unterschied z.B. zwischen Männern und Frauen als Effekt des Geschlechts interpretieren und geht dabei davon aus, dass sich mehr dahinter verbirgt, als wenn man rein zufällig zwei Gruppen bildet und dann vergleicht.

\subsection{Literatur}

Adams, R. \& Wu, M., (Hrsg.). (2002). PISA 2000 technical report. Paris: OECD.

Blömeke, S. (2011). Überzeugungen in der Lehrerausbildungsforschung. Wie lässt sich dasselbe in unterschiedlichen Kulturkreisen messen? Beiträge zur Lehrerbildung, 29(1), 53-65.

Blömeke, S., Kaiser, G. \& Lehmann, R. H. (2008). Professionelle Kompetenz angehender Lehrerinnen und Lehrer. Wissen, Überzeugungen und Lerngelegenheiten deutscher Mathematikstudierender und -referendare. Erste Ergebnisse zur Wirksamkeit der Lehrerausbildung. Münster: Waxmann.

Blömeke, S., Lehmann, R. H. \& Suhl, U. (2010). Technischer Anhang zu TEDS-M 2008 Primarstufe: Stichprobenziehung, Durchführung der Erhebung, Skalierung, Gewichtung und Analyseeinheiten. In S. Blömeke, G. 
Kaiser \& R. H. Lehmann (Hrsg.). Professionelle Kompetenz und Lerngelegenheiten angehender Primarstufenlehrkräfte im internationalen Vergleich (S. 327-391). Münster: Waxmann.

Brandt, S., Oser, F., Biedermann, H., Kopp, M., Krattenmacher, S., Brühwiler, C. \& Steinmann, S. (2010). Country Report about Teacher Training in Switzerland. In: Lawrence Ingvarson \& Jack Schwille (Hrsg.). National Polices and Regulatory Arrangements for the Mathematics Preparation of Future Teachers in 18 Countries. Volume One of the Teacher Education and Development Study in Mathematics (TEDS-M). Amsterdam: IEA.

Dumais, J. \& Meinck, S. (2013a). Estimation weights, participation rates, and sampling error. In M. T. Tatto (Hrsg.). The Mathematics Teacher Education Study (TEDS-M): Technical Report (S. 129-159). Amsterdam: IEA.

Dumais, J. \& Meinck, S. (2013b). Sampling Design. In M. T. Tatto (Hrsg.). The Mathematics Teacher Education Study (TEDS-M): Technical Report (S. 79-91). Amsterdam: IEA.

Masters, G. N. (1982). A rasch model for partial credit scoring. Psychometrika, 47(2), 149-174.

Rasch, G. (1960). Studies in mathematical psychology: I. Probabilistic models for some intelligence and attainment tests. Copenhagen: The Danish Institute of Educational Research.

Schmidt, W., Tatto, M. T., Bankov, K., Blömeke, S., Cedillo, T., Cogan, L. et al. (2007). The preparation gap: Teacher education for middle school mathematics in six countries (MT21 Report). East Lansing, MI: Michigan State University.

Tatto, M. T., Schwille, J., Senk, S. L., Ingvarson, L., Peck, R. \& Rowley, G. (2008). Teacher Education and Development Study in Mathematics (TEDS-M). Policy, practice, and readiness to teach primary and secondary mathematics. Conceptual framework. Amsterdam: IEA.

Tatto, M. T., Schwille, J., Senk, S. L., Ingvarson, L., Rowley, G., Peck, R. et al. (2012). Policy, practice, and readiness to teach primary and secondary mathematics in 17 countries: Findings from the IEA Teacher Education and Development Study in Mathematics (TEDS-M). Amsterdam: IEA. 